\title{
Sink or swim: accumulation of biomass at fronts
}

\author{
Peter J. S. Franks \\ College of Oceanography, Oregon State University, Ocean Admin Bldg. 104, Corvallis, Oregon 97331-5503, USA
}

\begin{abstract}
The dense concentrations of biomass commonly seen at fronts are often explained by a physiological response of the organisms to the frontal environment. However, some fraction of the enhanced biomass may be explained through purely physical processes: the interaction of floating, sinking or swimming with the flows at a front. To explore this hypothesis, I present a model of steady, 2-dimensional cross-frontal circulations, which I combine with a variety of swimming behaviors. The models show how patchiness may arise at fronts through retention and accumulation zones, and the patterns which evolve given various flow and swimming characteristics. The ecological significance of the results is discussed.
\end{abstract}

\section{INTRODUCTION}

Numerous studies have shown oceanic fronts to be the sites of enhanced biomass. Tidally generated fronts around the British lsles are often the sites of massive dinoflagellate blooms (Pingree et al. 1975, Simpson et al. 1979), and show characteristic chlorophyll a patches associated with the frontal zone (Holligan 1981). At fronts formed by wind-driven upwelling, dense phytoplankton concentrations often develop in and around the uplifted pycnocline (Jones \& Halpern 1981, Dengler 1985, Traganza et al. 1987). Topographic fronts, formed in the lee of islands and headlands, are often the location of enhanced primary and secondary production (Simpson et al. 1982, Townsend et al. 1983). Water mass fronts and buoyant plume fronts are also commonly found to exhibit an enhanced biological biomass compared to surrounding waters (Houghton \& Marra 1983, Richardson 1985). The processes invoked to account for this enhanced production are diverse, and generally involve a physiological response of the organism to the physical dynamics of the front. Thus it is often hypothesized that the enhanced biomass is a result of increased nutrient uptake, increased growth rate, depressed photosynthetic response, etc., forced by a physical process. However, physiological responses may not be a sufficient explanation of the enhanced biomass.
Many of the organisms which display enhanced biomass at fronts also have the ability to float, sink or swim. If this movement allows the organism to deviate from the paths which the water particles follow, i.e. the organism is not a passive tracer, then the potential exists for the organism to become concentrated in certain types of flow. Thus some enhancement of biomass could be caused by a directed swimming behavior within the dynamic frontal region, independent of the physiological responses of the organism (e.g. Olson \& Backus 1985).

Such a hypothesis has been explored by several researchers, concentrating mainly on Langmuir circulations and internal waves. The theoretical work of Stommel (1949) and Evans \& Taylor (1980) examining the patterns created by particles sinking in Langmuir cells was supported by laboratory experiments of Watanabe \& Harashima (1986). These experiments showed that the flagellate Heterosigma akashiwo would accumulate in laboratory-generated circulation cells, giving enhanced concentrations independent of growth. Kamykowski (1981) showed that swimming dinoflagellates could interact with internal waves to create locally enhanced concentrations within the wave field. No studies, however, have examined the effects of a variety of swimming behaviors in a more general 2-dimensional flow.

In this paper I use a simple 2-dimensional model of steady cross-frontal flow to explore the mechanisms by 
which horizontal and vertical patchiness of biomass are generated as organisms float, sink and swim at fronts. I first define a general model, state its assumptions, and indicate the conditions under which various types of swimmers may generate patchiness. I then define the mechanisms by which this patchiness evolves (accumulation/retention zones). Finally I explore a specific model of cross-frontal flow, and examine the effects of various swimming behaviors on the organisms' positions and concentration.

\section{GENERAL MODEL}

In this section I will define the basic model assumptions, and derive some general results concerning the interaction of swimming organisms and physical flows. As will be demonstrated below, many of the conclusions reached in this section will be independent of the specifics of the flow or the swimming behavior, and thus are widely applicable. The variables used in the model are summarized in Table 1 The subscript ' $p$ ' on a variable refers to processes driven purely by the physics of the front, e.g. the frontal velocities. The subscript ' $s$ ' indicates a swimming function, while the subscript 'b' indicates a biological variable. The biological variables are usually a function of both physical and swimming processes. While swimming is the result of physiological responses and processes, the end product is a physical displacement; thus swimming will be referred to as a physical process in the present context.

A definition sketch of a hypothetical front is given in Fig. 1. Fronts are formed at the abutment of 2

Table 1. Definitions of variables used in the models

\begin{tabular}{|ll|}
\hline$C$ & Biological concentration field \\
$C_{0}$ & Initial concentration field \\
$h$ & Vertical scale of flow \\
$k$ & Parameter controlling strength and direction of flow \\
$R$ & Horizontal scale of flow \\
$t$ & Time \\
$t_{\mathrm{o}}$ & Initial time \\
$u_{\mathrm{b}}$ & Biological horizontal velocity \\
$u_{\mathrm{p}}$ & Physical horizontal velocity \\
$u_{\mathrm{s}}$ & Horizontal swimming velocity $\{=0\}$ \\
$w_{\mathrm{b}}$ & Biological vertical velocity \\
$w_{\mathrm{o}}$ & Maximal swimming speed \\
$w_{\mathrm{p}}$ & Physical vertical velocity \\
$w_{\mathrm{s}}$ & Swimming velocity \\
$x$ & Horizontal coordinate \\
$z$ & Vertical coordinate \\
$z_{\mathrm{o}}$ & Depth at which swimming speed is minimum or zero \\
$z_{\mathrm{w}}$ & Vertical length scale of swimming function \\
$\theta$ & Angle of front to surface \\
$\psi_{\mathrm{b}}$ & Biological stream function \\
$\psi_{\mathrm{p}}$ & Physical stream function
\end{tabular}

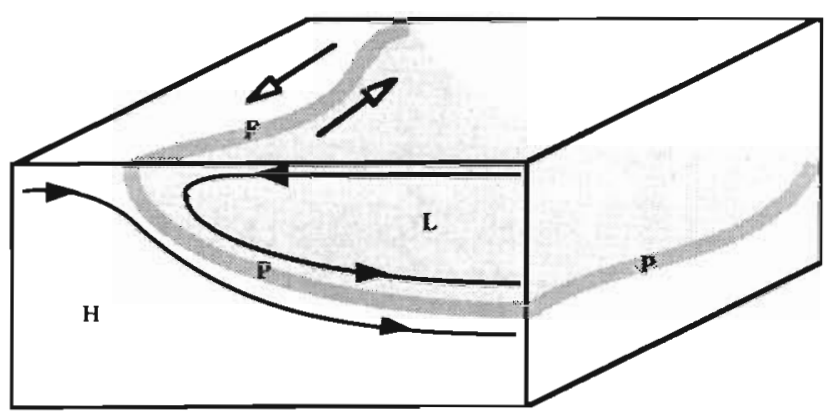

Fig. 1. Definition sketch of a hypothetical front. Open arrows: along-frontal flows assuming a quasigeostrophic balance; filled arrows: cross-frontal flows. The light (L) and heavy $(\mathrm{H})$ water masses are indicated, as well as the pycnocline $(\mathrm{P})$ and the front $(F)$

dissimilar water masses, usually light (warm, fresh) and heavy (cold, salty). The front itself is characterized by an upward or downward curving of the pycnocline, such that the pycnocline intersects the surface or bottom. This gives a strong horizontal density gradient in the direction perpendicular to the front (the crossfrontal direction). Properties tend to be relatively uniform along the front itself, where there are generally strong along-front flows. The cross-frontal flows are much weaker; however, it is the influence of these flows on swimming organisms which will be explored in the present model.

Consideration of flows in the present study will be restricted to steady 2 -dimensional flows which can be described by a stream function, $\psi_{\mathrm{p}}=\psi_{\mathrm{p}}(x, z)$, where $x$ is the cross-frontal coordinate and $z$ is depth. The flow thus has no time-dependence, and does not include the effects of mixing (but see Olson \& Backus 1985). These assumptions are quite restrictive, but allow for simple analytic solutions to the model equations. It will also be assumed that the swimming behavior of the organisms is restricted to vertical motions, i.e. $w_{\mathrm{s}}=w_{\mathrm{s}}(z), u_{5}=0$, where $w_{\mathrm{s}}(z)$ is the vertical swimming speed of the organism as a function of depth. This assumption may be justified in 2 ways. First, the ratio of the horizontal to vertical scales of the front is often 1001 i thus an organism would have to swim 100 times farther horizontally to have the same relative displacement as a vertical motion. Second, swimming behaviors are generally directed by a gradient in some property (light, temperature, nutrients, particle concentration, etc.) The gradients in these properties are far stronger vertically than horizontally, thus the stimulus for directed horizontal swimming is weak.

The stream function, $\psi_{\mathrm{p}}$, gives the paths which the water particles follow. From the stream function, the physically induced horizontal and vertical velocities, $u_{\mathrm{p}}$ and $w_{\mathrm{p}}$ can be found: 


$$
\begin{aligned}
& u_{p}=\frac{\partial \psi_{p}}{\partial z} \\
& w_{p}=-\frac{\partial \psi_{p}}{\partial x} .
\end{aligned}
$$

These are the velocities of water particles, generated through the physical processes which created the flow

When we consider organisms swimming in a vertical flow, there are 2 cases which may arise: weak swimmers and strong swimmers. Here weak swimmers are defined as those organisms whose vertical swimming speed is affected by the ambient vertical water velocity. For a weak swimmer with swimming speed $w_{\mathrm{s}}$, the resultant vertical velocity, $w_{b}$, will be:

$$
w_{\mathrm{b}}=w_{\mathrm{s}}+w_{\mathrm{p}}
$$

Thus weak swimmers have a vertical velocity, $w_{b}$, which is the sum of the swimming velocity and the ambient vertical velocity.

Strong swimmers are defined as those organisms whose vertical velocity, $w_{b}$, is not affected by the ambient vertical water velocities, thus:

$$
w_{\mathrm{b}}=w_{\mathrm{s}}
$$

For both strong and weak swimmers, the horizontal component of motion, $u_{\mathrm{b}}$, is assumed to be driven purely by the ambient physical motions, thus:

$$
u_{\mathrm{b}}=u_{\mathrm{p}}
$$

The distinction between strong and weak swimmers lies in the strength of their swimming compared to the ambient vertical water velocities. Vertical velocities at fronts are notoriously difficult to measure, but estimates from field studies and modelling indicate maximal speeds of ca $0.2 \mathrm{~mm} \mathrm{~s}^{-1}$ (Foo 1981, Smith et al. 1983, Chao 1987, Werner 1987). This is the same order of magnitude as the swimming speed of most ciliates, flagellates, dinoflagellates, coccolithophorids, and diatoms (for example Bauerfeind et al. 1986); thus these organisms are considered weak swimmers in the present model. Most crustaceans (copepods, euphausiids, amphipods, etc.) as well as many types of fecal pellets have vertical velocities 1 or 2 orders of magnitude higher than the vertical velocities at fronts. These, then, are strong swimmers (sinking is considered 'swimming' for the present context).

If we define a concentration field of organisms, $C=C(x, z, t)$, the equation of continuity in this 2 dimensional system is (e.g. Batchelor 1983, p. 74):

$$
\frac{\partial C}{\partial t}=-\left(\frac{\partial}{\partial X}\left(C u_{\mathrm{b}}\right)+\frac{\partial}{\partial z}\left(C w_{\mathrm{b}}\right)\right)
$$

Thus the rate of change of organism concentration at a point is given by the spatial gradients of the product of the concentration and the velocity. More specifically, this equation defines the rate of accumulation or loss of particles at a point through purely physical processes: 2-dimensional frontal flows, and swimming. Thus patchiness will develop if there is any spatial dependence to $\frac{\partial C}{\partial t}$.

For weak swimmers, we can include Eqs. (2) \& (4) in Eq. (5), and make use of Eq. (1) to get:

$$
\frac{\partial C}{\partial t}=-\left(u_{\mathrm{p}} \frac{\partial C}{\partial x}+w_{\mathrm{p}} \frac{\partial C}{\partial z}+\frac{\partial}{\partial z}\left(C w_{\mathrm{s}}\right)\right)
$$

This equation describes the physically induced spatial patchiness of weak swimmers in a general 2dimensional flow. In the special case when the initial concentration of organisms, $C$, has no horizontal dependence, i.e. $C\left(x, z, t_{0}\right)=C_{0}(z)$, we find from Eq. (6) that:

$$
\frac{\partial C}{\partial t}=-\left(w_{\mathrm{p}} \frac{\partial C}{\partial z}+\frac{\partial}{\partial z}\left(C w_{\mathrm{s}}\right)\right)
$$

The only variable which has any horizontal dependence in this equation is $w_{p}$, the ambient vertical velocity. This gives the somewhat surprising result that initially all the horizontal dependence of the weak swimmer's concentration field is generated by the horizontal structure of $w_{p}$, the physically induced vertical velocity. In other words, under these assumptions, the horizontal patchiness of a weak swimmer should initially have the same horizontal scale as the vertical velocity.

For strong swimmers, we can substitute Eqs. (3) \& (4) in Eq. (5) to get:

$$
\frac{\partial C}{\partial t}=-\left(u_{p} \frac{\partial C}{\partial X}+C \frac{\partial u_{p}}{\partial x}+\frac{\partial}{\partial z}\left(C w_{s}\right)\right) .
$$

Eq. (8) describes the physical processes leading to patchiness of strong swimmers in a steady, 2dimensional flow. Note that there are no terms containing $w_{p}$, the ambient vertical velocity. If we again make the assumption that $C\left(x, z, t_{0}\right)=C_{0}(z)$ only, then from Eq. (8) we find:

$$
\frac{\partial C}{\partial t}=-\left(C \frac{\partial u_{p}}{\partial x}+\frac{\partial}{\partial z}\left(C w_{s}\right)\right) .
$$

The only term with a horizontal dependence in Eq. (9) is $\frac{\partial u_{p}}{\partial x}$, the horizontal gradient of the horizontal velocity. Again, this is a rather surprising result, stating that initially, the horizontal patchiness of strong swimmers is controlled by the horizontal gradient of the 
horizontal velocity. Thus the mechanisms underlying the initial horizontal patchiness of weak and strong swimmers are quite distinct. For both weak and strong swimmers, as the biological patterns develop, the term $u_{\mathrm{p}} \frac{\partial C}{\partial \mathrm{x}}$ will become a more important component generating horizontal patchiness.

The results obtained above are dependent only on the assumptions stated above: steady 2-dimensional flow, and vertical swimming. The results do not depend on the structure of the flow, or the vertical dependence of the swimming. Thus these results are quite general, and applicable to a wide variety of oceanic environments.

\section{ACCUMULATION, RETENTION ZONES}

The analysis presented above demonstrated that patchiness can be generated by organisms swimming vertically in a steady 2 -dimensional flow. However, the mechanisms by which this patchiness is created were not defined. Here I explore the actual processes which result in patchiness in this physical/biological system.

The generation of patchiness occurs through 2 basic mechanisms: retention and accumulation. A retention zone is characterized by closed particle paths, i.e. organisms cycle continuously about a closed orbit. Accumulation zones are characterized by convergent particle paths: organisms are continuously advected toward a region and become concentrated (Fig. 2).

The distinction between retention and accumulation zones is important: there is no physically induced change in amount of biomass in a retention zone. Neglecting biological terms such as growth, grazing or death, the number of organisms within the retention zone would be the same as the initial number of organisms. If organisms are lost from the region surrounding the retention zone, the zone would appear as a patch of enhanced biomass. However, the number of organisms within the retention zone has not changed since there is no gain or loss of particles from a retention zone.

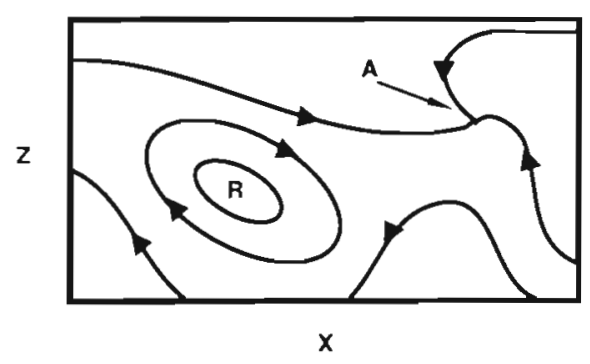

Fig. 2. Retention (R) and accumulation (A) zones in a hypothetical flow. Retention zones are characterized by closed, and accumulation zones by convergent particle streamlines
This is not true of an accumulation zone. Organisms are advected into an accumulation zone, where they slow, or stop moving relative to the front. Thus their concentration is continuously increasing through purely physical processes: vertical swimming in the ambient flow. Two types of accumulation zone are important in physical/biological interactions; I will term these compression and convergence zones (Fig. 3). Compression occurs as organisms move into a region faster than they move out, creating a net accumulation of biomass. Convergence occurs as organisms are advected toward a region where they stop moving relative to the front. The important difference between compression and convergence is that organisms continuously move through a compression zone, whereas they stop in a convergence zone. Thus the residence time of organisms is limited in a compression zone, but infinite in a convergence zone. In a steady flow, a patch created at a compression zone may move with the flow, while a patch at a convergence zone will remain stationary. Examples of both compression and convergence zones will be given below.

In terms of the model presented above, an accumulation (compression or convergence) zone occurs if $\frac{\partial C}{\partial t}>0$, i.e. an increase in biomass due to a convergence in the biological flow. From Eqs. (6) \& (8), this convergence will arise from both gradients in the concentration field $C(x, z, t)$, and gradients in the swimming velocity, $\frac{\partial w_{5}}{\partial z}$. Ignoring, for the moment, contributions from gradients in the concentration field, for weak swimmers accumulation zones will develop if $\frac{\partial w_{s}}{\partial z} \neq 0$. That is, patchiness will develop if there is a vertical dependence to the swimming behavior. For strong swimmers, if $\frac{\partial w_{\mathrm{s}}}{\partial z} \neq \frac{\partial u_{p}}{\partial x}$, then accumulation zones will occur. Both these situations are likely to arise in nature.

\section{ACCUMULATION}

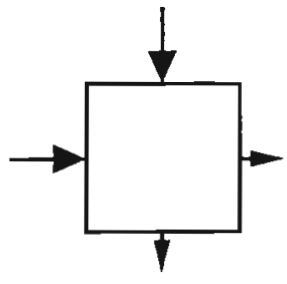

COMPRESSION

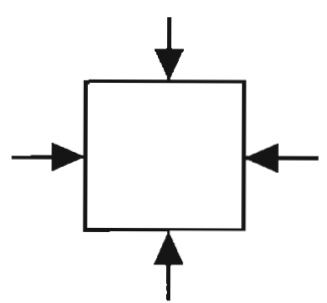

CONVERGENCE
Fig. 3. Two types of accumulation zone: compression and convergence. The size of the arrow is proportional to the flux of biomass across the indicated face of the box 
The opposite of an accumulation zone is a dispersal zone, defined by $\frac{\partial C}{\partial t}<0$. Here organisms are actively being lost, and their concentration is decreasing. Dispersal zones occur under the same circumstances as accumulation zones.

Retention zones are less easy to define in terms of this model, and will arise only in restricted cases (Stommel 1949, Evans \& Taylor 1980). If it is possible to define a stream function for the organisms, $\psi_{b}$, then a retention zone occurs at a local extremum of $\psi_{b}$, i.e.

$$
\frac{\partial \psi_{\mathrm{b}}}{\partial x}=\frac{\partial \psi_{\mathrm{b}}}{\partial z}=0
$$

In general, however, $\psi_{b}$ cannot be defined. An interesting result from Stommel (1949) is worth restating in terms of the present model: compression zones can occur within retention zones to give variations in concentration inside the retention zone. However, as noted above, the total amount of biomass in the retention zone remains unchanged by these processes.

\section{FRONTAL MODEL}

Using a simple model I have demonstrated that patchiness can arise through the interaction of weak and strong swimmers with steady 2-dimensional flows. I have described how this patchiness can arise via retention and accumulation zones, based on the swimming behavior and flow structure. I will now use a specific model of steady 2-dimensional cross-frontal flows to explore the patterns of patchiness which arise under various types of swimming behavior. The results obtained will be less general than those obtained above, but should be applicable to a range of frontal types.

The stream function for the cross-frontal circulation, $\psi_{\mathrm{p}}(X, Z)$, will be given by the following function

$$
\psi_{\mathrm{p}}(x, z)=k z \sin \theta \tanh \left(\frac{x}{R}+\frac{z}{h} \cos \theta\right) .
$$

Again, $x$ is the cross-frontal and $z$ the vertical coordinate. The parameter $k$ can be thought of as the maximal horizontal velocity away from the front (units: $\mathrm{m} \mathrm{s}^{-1}$ ), and controls the strength and direction of the flow. For $k<0$, the flow will be convergent, while $k>0$ gives divergent flow (Fig. 4). $R$ gives the horizontal scale of the front, and can be considered similar to the internal Rossby radius of deformation. The vertical scale is $h$, which could be the depth of the pycnocline away from the front. The parameter $\theta$ is the angle the front makes with the horizontal: if $\theta=90^{\circ}$, the flow at the front will be directed vertically (upwards or downwards), and the front is symmetric. If $\theta=45^{\circ}$, the flow will angle to the side, giving a sloping front with a pro-
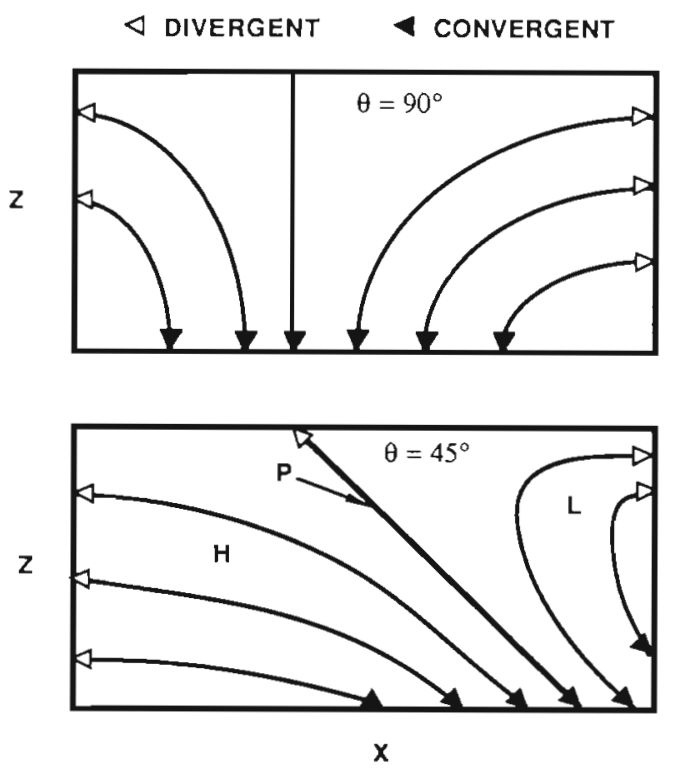

Fig. 4. Two cases of the model stream function $\left(\psi_{b}\right)$. Filled arrows: direction of convergent flow $(k<0)$; unfilled arrows direction of divergent flow $(k>0)$. Upper panel: $\theta=90^{\circ}$; lower panel: $\theta=45^{\circ}$ The pycnocline (P), light (L) and heavy $(H)$ water masses are shown

nounced asymmetry (Fig. 4). It is this asymmetry in the flow which leads to asymmetric distributions of plankton about the front. The ambient horizontal and vertical velocities, $u_{p}$ and $w_{p}$, are found from Eq. (1).

The functional form of $\psi_{p}$ given by Eq. (11) was chosen somewhat arbitrarily, but gives a flow structure qualitatively similar to those suggested by Pingree et al. (1974), James (1978), Simpson et al. (1978), Foo (1981), Garrett \& Loder (1981) and Chao (1987). These studies were performed at tidal, wind-driven and buoyancy-driven fronts; thus the present model should be widely applicable. It is not difficult to show that the patterns of patchiness which will be described below do not depend very strongly on the details of the steady flow. Many different functional forms for $\psi_{\mathrm{p}}$ could have been used with similar results, suggesting that the model is quite robust.

For ease of describing the results, it is necessary to define some terminology before proceeding. With reference to Fig. 4, the pycnocline (P) will be defined as the region of maximal vertical flow, i.e. the boundary separating 2 flow cells. The 'light' (L) side of the front will refer to the flow cell which is closest to the surface, while the 'heavy' $(\mathrm{H})$ side of the front refers to the deeper flow cell. This terminology is chosen to reinforce the idea of a 2 -layered fluid separated by a sloping front, with the flows confined to the waters of each layer (no diapycnal flows). It is not necessary, however, to restrict the interpretation of the results to flows under these particular circumstances. 
The solutions to the equations will be shown as both particle positions and particle paths. A matrix of $62 \times 44(x, z)$ particles was followed for a time of $R / 2 \mathrm{k}$. For a front of horizontal scale $R=5 \mathrm{~km}$ and $\mathrm{k}=0.5 \mathrm{~m} \mathrm{~s}^{-1}$, this gives a time $R / 2 \mathrm{k}$ of ca $0.06 \mathrm{~d}$. The initial particle field and water streamlines are shown in Fig. 5, for both divergent and convergent flows (refer to Fig. 4 for definition of 'divergent' and 'convergent'). The particle positions give an indication of compression and convergence zones. However, to distinguish the two, and to identify retention zones, it is necessary to refer to the particle paths. Thus particle positions are plotted for both weak and strong swimmers, while particle paths are plotted only for the weak swimmers for most of the swimming behaviors. The maximal swimming velocities used are equal to the maximal ambient vertical velocities at depth $z=-5$ (nondimensional depth). All solutions will be given for a front of $45^{\circ}$

\section{No swimming}

A variety of swimming behaviors, $w_{\mathrm{s}}(z)$ can be incorporated into this model. The most simple is zero swimming velocity:

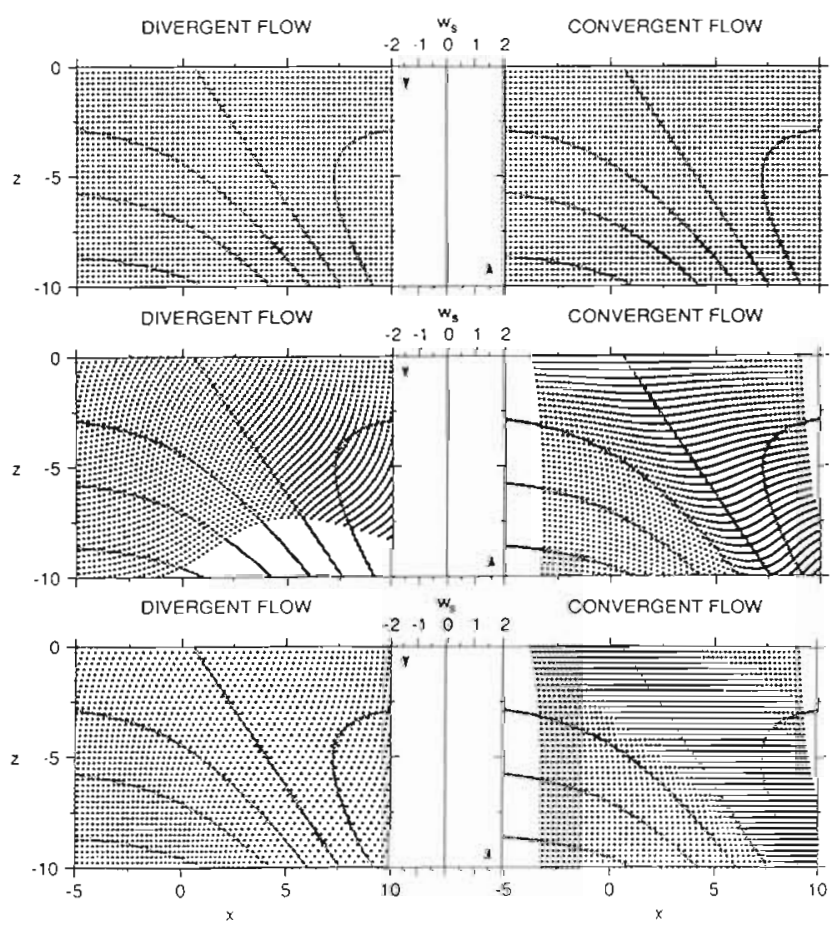

Fig. 5. No swimming. Upper row: initıal condition for particle positions. Middle row: weak swimmers' positions after a time $R / 2 \mathrm{k}$. Bottom row: strong swimmers' positions after $R / 2 \mathrm{k}$. Left column: divergent flow. Right column: convergent flow (see Fig. 4). Central column: swimming behavior, $w_{s}(z)=0$. Frontal flows $\left(\psi_{b}\right)$ shown by thick stippled lines

$$
w_{\mathrm{S}}=0
$$

This swimming behavior makes the differences between the weak and strong swimmers most apparent: weak swimmers become passive tracers and follow the water streamlines, while strong swimmers maintain their original depth $\left(w_{\mathrm{s}}=-w_{\mathrm{p}}\right)$, and are swept toward a convergent front, and away from a divergent front by the horizontal velocities (Fig. 5). Thus there is no change in density of weak swimmers. It may appear that the weak swimmers accumulate near a convergent front; however any horizontal compression of the particles is compensated for by a vertical stretching, and thus the particle concentration remains unchanged. Strong swimmers accumulate on the light side of a convergent front, and away from a divergent front.

\section{Sinking}

The next behavior to consider is simple sinking:

$$
w_{\mathrm{s}}(z)=-w_{0}
$$

where $w_{0}$ is the sinking velocity. The results for weak and strong swimmers are shown in Fig. 6 for both
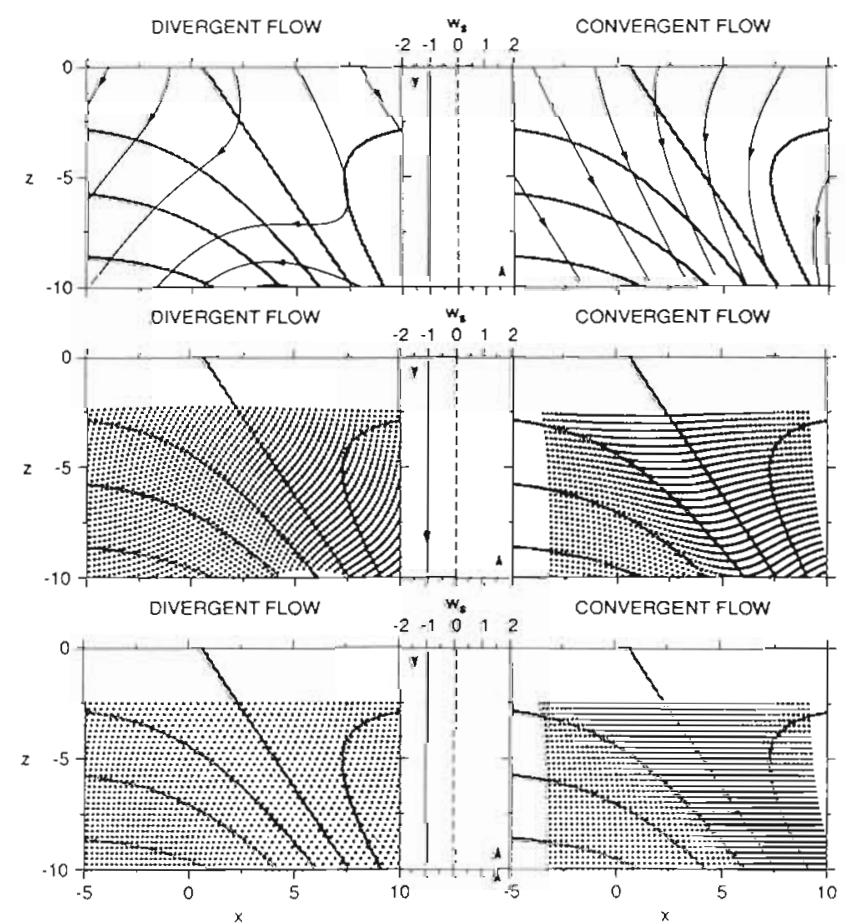

Fig. 6. Sunking. Upper row: thin black lines are weak swimmers' paths. Middle row: weak swimmers' positions after a time $R / 2 \mathrm{k}$. Bottom row: strong swimmers' positions after $R / 2 \mathrm{k}$. Left column: divergent flow. Right column: convergent flow (see Fig. 4). Central column: swimming function, $w_{\mathrm{s}}(z)$. Frontal flows $\left(\psi_{b}\right)$ shown by thick stippled lines. All units nondimensional 
divergent and convergent flows. From the particle paths for the weak swimmers, it can be seen that organisms or fecal pellets sinking from the light side of a divergent front will cross the front, and be swept to the heavy side. Weak swimmers within the pycnocline of a divergent front are swept upwards before sinking out of the flow toward the heavy side of the front. There is no change in concentration of weak swimmers, as shown by the particle positions. Strong swimmers, however, show strong dispersal rates at the pycnocline of a divergent front.

The situation is almost reversed for a convergent front. Weak swimmers sinking in a convergent front tend to be swept toward the pycnocline on both the light and heavy sides of the front, with some horizontal compression at the pycnocline. Strong swimmers show high accumulation rates in a compression zone on the light side of the convergent front.

\section{Floating}

Floating organisms have a constraint which sinking organisms do not: they must have zero vertical velocity at the surface. This boundary condition leads to more complicated patterns of accumulation than the case of simple sinking. I define a floating velocity, $w_{s}(z)$ as:

$$
w_{\mathrm{s}}(z)=w_{0} \tanh \left(\frac{z}{z_{w}}\right) .
$$

where $w_{0}$ is the maximal floating speed, and $z_{w}$ the length scale determining the region in which the particle slows down toward the surface. Large $z_{w}$ describes cells slowing down farther from the surface than small $Z_{w}$. Results of the model using this floating behavior are shown in Fig. 7. In a divergent flow, both weak and strong swimmers accumulate at the surface, near the front, with slightly higher rates on the light side of the front. The results are much more asymmetric at a convergent flow, with the highest accumulation rates at a convergence zone at the surface on the light side of the front. The particle paths for the weak swimmers show organisms crossing the front from the heavy to the light side, while being forced downward at the convergent front. Strong swimmers are similarly swept toward the front at the surface, but are not forced downward by the flow at depth.

\section{Depth-directed swimming}

The function describing the floating of organisms can be generalized to describe the swimming of organisms toward a particular depth, $z_{0}$ :

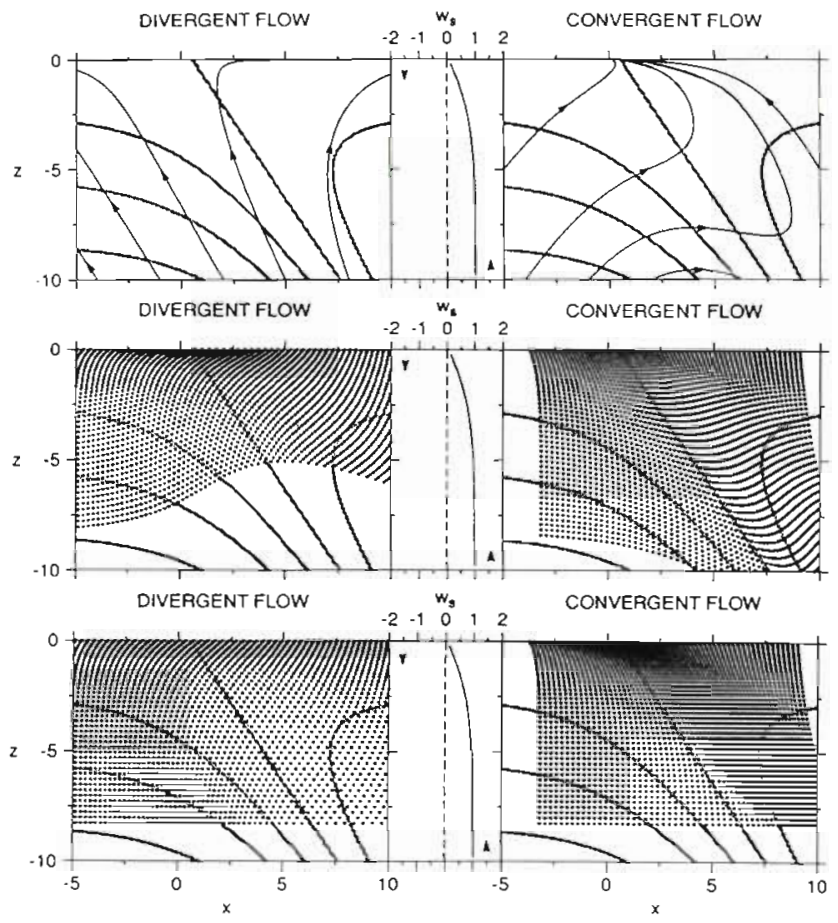

Fig. 7. Floating. Legend same as Fig. 6

$$
w_{\mathrm{s}}(z)=w_{0} \tanh \left(\frac{z-z_{0}}{Z_{w}}\right)
$$

All the parameters are the same as the floating model. This function describes organisms swimming downward from the surface, and upward from depth, toward depth $z_{0}$. This depth $z_{0}$ could represent an isolume, isotherm, etc. The sinking and floating functions described above (Eqs. 13 \& 14) are actually specific cases of this function (Eq. 15).

This depth-directed swimming model gives very asymmetric patches, as shown in Fig. 8. In a divergent flow, weak swimmers accumulate in a compression zone at the front, while strong swimmers are dispersed, accumulating away from the front at depth $z_{0}$. In a convergent flow, the organisms are strongly concentrated at a convergence zone on the light side of the front. Weak swimmers also show compression zones near depth $z_{0}$ on both sides of the convergent front. The convergence zone extends away from the front toward the light side, with weak swimmers being swept to a greater depth than strong swimmers. Both weak and strong swimmers show a tilting of the accumulation zone in the direction of the front.

\section{Vertical migration}

Vertical migration is a swimming behavior displayed by a wide variety of organisms in the ocean. To simu- 


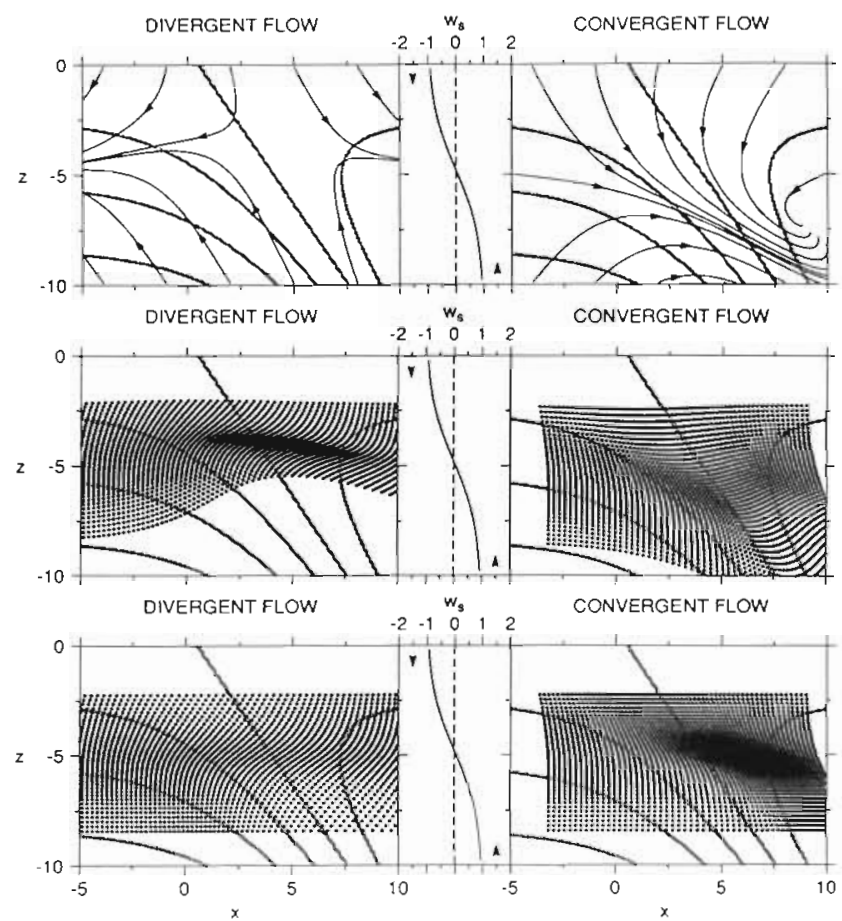

Fig. 8. Depth-directed swimming. Legend same as Fig. 6

late vertical migration through the model front, I combined the sinking and floating behaviors (Eqs. $13 \& 14$ ) in modeis of weak and strong swimmers. The $62 \times 44$ $(x, z)$ matrix of particles was followed as they swam downward for $2 R / k$, upward for $4 R / k$, and then downward for $2 R / k$. For a front with horizontal scale $R=$ $5 \mathrm{~km}$, and maximal horizontal velocities of $k=$ $0.5 \mathrm{~m} \mathrm{~s}^{-1}$, the total migration time, $8 R / k$, is approximately $1 \mathrm{~d}$. The results of the model over 1 full migration period are shown in Fig. 9 for weak swimmers and Fig. 10 for strong swimmers in both divergent and convergent flows.

As the migrators move downward, the weak swimmers are compressed vertically at the pycnocline in a divergent flow, while the strong swimmers are dispersed by horizontal divergences. At a convergent flow, both weak and strong swimmers are horizontally compressed toward the pycnocline. The weak swimmers also become stretched along the pycnocline by the vertical flows

During the upward phase of the migration, weak swimmers in a divergent flow become more vertically compressed at the front, while experiencing a horizontal shear which reduces the number of organisms at the front. Strong swimmers become further dispersed at the front, but do not experience the vertical compression of the weak swimmers. Some increase in concentration is found at the surface for both weak and strong swimmers, since the surface forms a

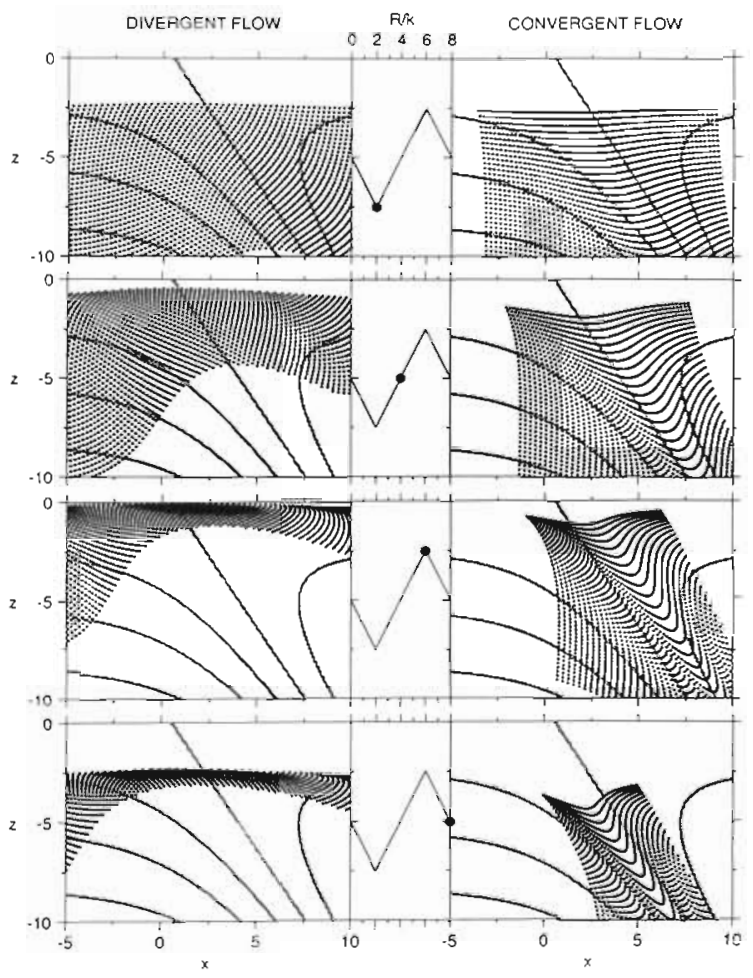

Fig. 9. Vertical migration: weak swimmers. Left column: divergent flow; right column: convergent flow. Central column: $(\bullet)$ relative vertical position in the migration cycle at the indicated time (units of $R / k$ ). The organisms swim downward for the first $2 R / k$ (top row of panels), upward for the next $4 R / k$ ( 2 middle rows), and downward for the last $2 R / k$ (bottom row)

barrier to migration. The compression of weak swimmers at the front by the vertical velocities leads to much higher organism concentrations than the strong swimmers.

Organisms migrating upward in a convergent flow become increasingly compressed toward the pycnocline. Strong swimmers show very high concentrations at the surface due to vertical compression by swimming and horizontal compression caused by the flow. This is seen to a lesser extent in the weak swimmers, which become dispersed along the pycnocline away from the front

In the final downward phase of the migration, weak swimmers at a divergent front form a thin, concentrated horizontal band at the front, with the bulk of the organisms away from the front. Strong swimmers do not become concentrated by the vertical flow at a front; thus the horizontal divergence of the flow field causes low concentrations at the front. In contrast, both weak and strong swimmers become highly concentrated at the pycnocline of a convergent front. The weak swimmers, however, show lower concentrations and are spread farther along the pycnocline than the strong swimmers 


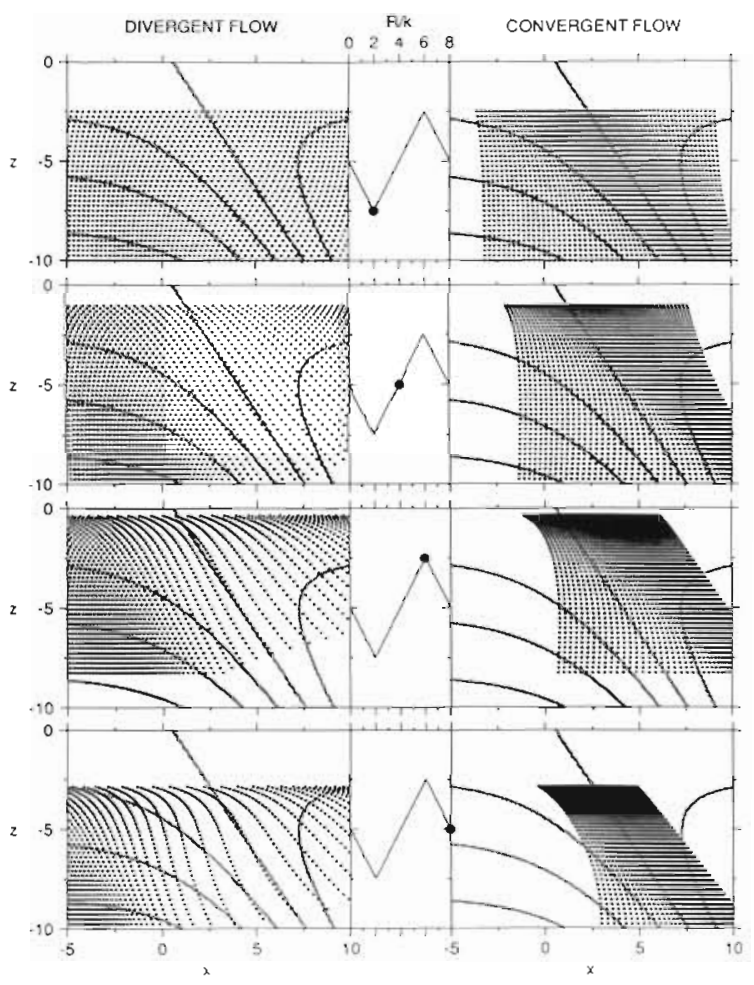

Fig. 10. Vertical migration: strong swimmers. Legend same as Fig. 9

\section{Swimming speed}

It is instructive to examine the effects of various swimming speeds on the patterns created by the organisms swimming at fronts. In Fig 11, the particle paths and particle postions of weak swimmers are plotted for maximal swimming speeds of $0.5 \times$ and $2 \times$ the maximal ambient vertical velocity at $z=-5$. The depthdirected swimming function (Eq. 15) was chosen since it includes both upward and downward swimming.

At the lowest swimming speeds, the organisms almost follow the water streamlines. They are swept away from the divergent front and toward the convergent front, but tend not to cross the front. The organisms are not strongly compressed, and the accumulation rates would be relatively low. As the swimming speeds increase, the swimmers cross the front, and strong accumulation zones develop near the pycnocline on the light side of the convergent front (convergence zone), and at the pycnocline of a divergent front (compression zone). The convergence zone is particularly pronounced at the convergent front, and moves upward toward depth $z_{0}$ with increasing swimming speeds. The accumulation rate also increases with swimming speed at both convergent and divergent fronts, as organisms concentrate at depth $z_{0}$. At a swimming speed of $2 \times$ the maximal ambient vertical
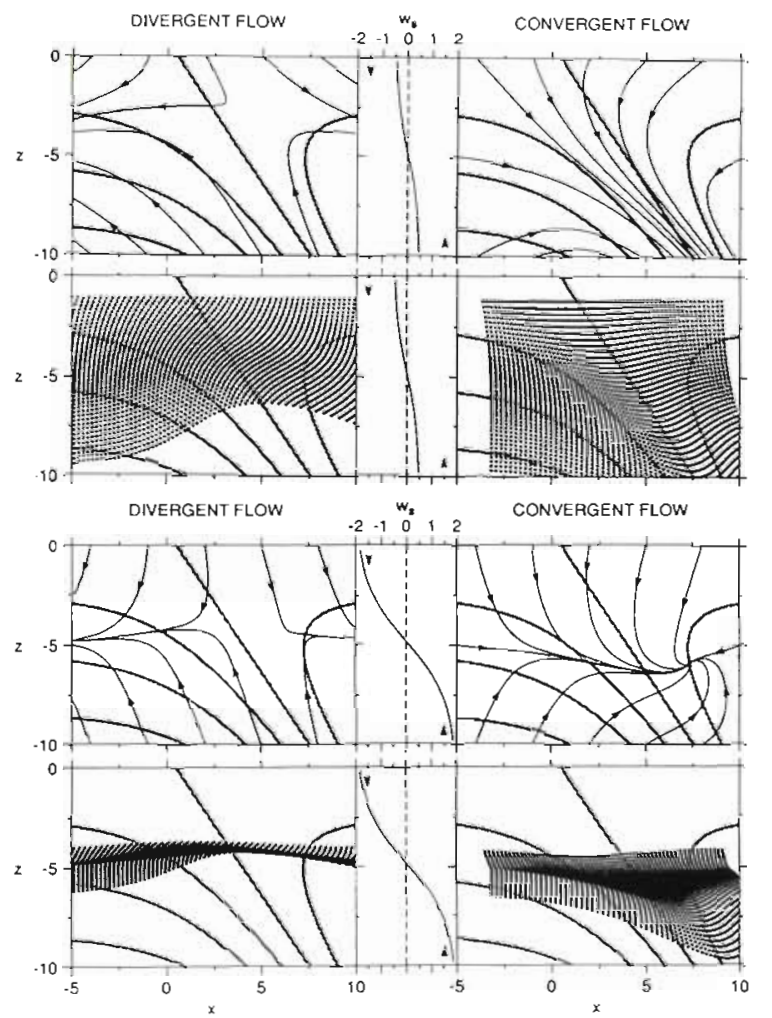

Fig. 11. Depth-directed swimming particle paths and positions for weak swimmers at 2 swimming speeds: 0.5 (upper 2 rows) and 2.0 (lower 2 rows) times the maximal ambient vertical velocity at $z=-5$

velocity, the swimming patterns are very similar to those of the strong swimmers (not shown). For both convergent and divergent fronts, stronger swimmers remain closer to the front than weak swimmers. The horizontal flows at a divergent front tend to sweep weak swimmers away from the front, while the strong vertical flows at a convergent front carry the organisms downward, along the pycnocline.

The actual rate of accumulation will vary depending on the swimming behavior, the swimming speed, and the ambient velocity structure. The maximal accumulation rate will occur at the point where $u_{\mathrm{b}}=w_{\mathrm{b}}=0$. For weak swimmers, this implies that $w_{\mathrm{s}}+w_{\mathrm{p}}=0$, and the biomass-specific rate of accumulation is given by:

$$
\frac{1}{C} \frac{\partial C}{\partial t}=-\frac{\partial w_{\mathrm{s}}}{\partial z}
$$

evaluated at the depth where $w_{\mathrm{s}}+w_{\mathrm{p}}=0$. For a weak swimmer using depth-directed swimming (Eq. 15) with a maximal speed of $0.1 \mathrm{~mm} \mathrm{~s}^{-1}$, and a vertical variation, $z_{\mathrm{w}}$, of $5 \mathrm{~m}$, the maximal accumulation rate is ca $2 \mathrm{~d}^{-1}$ (and will generally be much less). This is the same order as the maximal growth rates measured for most phytoplankton. 


\section{DISCUSSION}

Using a simple 2-dimensional model of steady crossfrontal flow combined with vertically-directed swimming, I have shown that horizontal and vertical patchiness can develop under a wide variety of circumstances. The horizontal and vertical scales of patchiness differ for weak and strong swimmers, and are controlled by both the swimming behavior and the ambient velocity structure. Weak swimmers are more influenced by the frontal velocity structure than strong swimmers; both types of swimmers are likely to create accumulation/dispersal zones around the front. The accumulation rates of swimmers at the front can be the same order as their maximal growth rates.

The patterns of accumulation of weak and strong swimmers vary in detail, but generally show accumulation along the pycnocline of convergent fronts, and in a horizontal band crossing a divergent front. These results are shown schematically in Fig. 12. The results of the vertical migration study (Figs. $9 \& 10$ ) are not very different than the sinking or floating studies (Figs. $7 \& 8)$. The longer time of integration $(8 R / \mathrm{k}$ versus $R / 2 \mathrm{k}$ ) allowed the patterns to evolve more fully, creating a concentrated patch along the pycnocline of a convergent front, and a concentrated horizontal layer of weak swimmers at a divergent front (cf. Fig. 12). The fact that the patterns are robust for different swimming
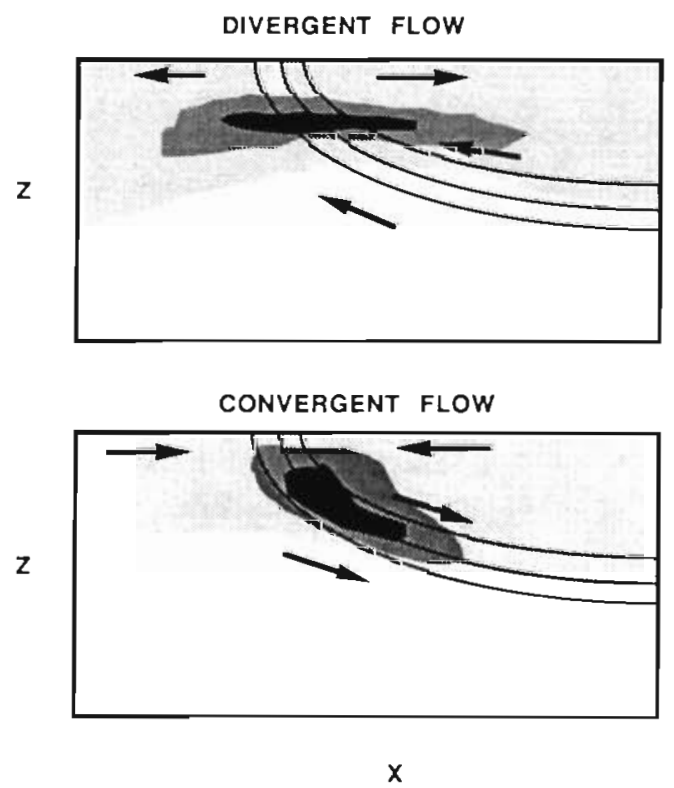

Fig. 12. Schematic summary of model results for organisms swimming in (upper) divergent and (lower) convergent flows. Stippling: different concentrations of a hypothetical phytoplankton population; thin lines: isopycnals. Arrows: direction of cross-frontal flow. Organisms are concentrated into a thin honzontal band crossing isopycnals at a divergent flow, and are concentrated along the pycnocline at a convergent flow behaviors, and combinations of swimming behaviors, suggests that the model may be simulating a fundamental process which should be identifiable in nature.

The patchiness patterns generated by the frontal model reflect the results of the general model: the patchiness of weak swimmers tends to be correlated with the ambient vertical velocity structure, while the patterns of strong swimmers are more closely tied to the horizontal gradient of the horizontal velocity. Thus weak swimmers tend to have high concentrations near the front, where the vertical velocities are greatest. In divergent flows this is manifested as a horizontal band, while in convergent flows the band follows the pycnocline; however the concentrations are highest at the front. Note, though, that the vertically integrated number of organisms may be lower at a divergent front than away from the front. In contrast, strong swimmers have low concentrations at a divergent front, as organisms are advected away from the front by the ambient horizontal velocities. This situation is reversed at a convergent front, where strong swimmers are compressed by the horizontal flows. Where these horizontal flows have their highest acceleration or deceleration is where the greatest dispersal or accumulation of strong swimmers occurs

The patchiness patterns generated by the model of swimmers at convergent fronts are qualitatively similar to the chlorophyll patches which are often recorded at convergent tidal fronts (e.g. Holligan 1981). These patches tend to be located within or slightly above the pycnocline, with the maximal concentrations below the surface. This is consistent with the results of the present model, which developed patches along the pycnocline, regardless of the swimming behavior at the convergent front (Fig. 12). Such patterns were also recorded by Tyler et al. (1982) for the dinoflagellate Gyrodinium uncatenum at a convergent front in the Chesapeake Bay. In this case the concentration and spreading of the motile cells along the convergent front coincided with a region of dense cyst concentrations in the sediment.

The accumulation patterns of motile phytoplankton at divergent (upwelling) fronts are predicted by the present model to be thin, concentrated horizontal layers at the front, with more diffuse cells away from the front (Fig. 12). This pattern is distinct from that seen at a convergent front in that it crosses isopycnals. Such patterns have been recorded for dinoflagellates at an upwelling front in the Chesapeake Bay, USA (Seliger et al. 1981), and in the upwelling fronts of the Skagerrak-Kattegat, Scandinavia (Richardson 1985). Thus the qualitative features of the present model show good agreement with extant data sets, suggesting that the basic model dynamics may be simulating real processes. 
It was shown that weaker swimmers tend to follow the water streamlines more closely than stronger swimmers (Fig. 11). Thus the weakest swimmers would be less concentrated, and would be advected along the pycnocline farther from a convergent front than stronger swimmers (regardless of swimming behavior). This would lead to a sorting of organisms along the pycnocline of the front, with the strongest swimmers near the front, and the weaker swimmers away from the front. Such a spatial dislocation of populations has been recorded at tidal fronts, where motile ciliates and flagellates dominated near the front, while diatoms were found farther from the front (Pingree et al. 1978, Simpson et al. 1979, Holligan 1981). Strong cross-frontal gradients in the planktonic community structure have also been recorded at winddriven upwelling zones, for example off Peru (Beers et al. 1971), and off the coast of Northwest Africa (Blasco et al. 1981). These gradients are generally acknowledged to be physically mediated, although the precise mechanisms have not been specified.

The retention zones discussed by Stommel (1949) and Evans and Taylor (1980) for Langmuir circulations are unlikely to arise in the open flow cells used in the present study. Evans and Taylor (1980) made the point that such zones are unlikely to exist even in Langmuir cells, due to variability in the flow and swimming behavior. Accumulation zones, however, are likely to appear whenever a swimming population consistently changes its swimming speed, such as tracking an isolume, isotherm, crossing a pycnocline, nutricline etc. The horizontal patchiness created in these situations depends on the swimming behavior and the ambient flows, as shown above.

In the models presented above, I have specifically ignored a growth response of the organisms. To include such a response would considerably restrict the applicability of the models, since a space or timedependent growth function would have to be specified. However, some intuitive idea of the physiological response of the organisms to their new environment can be obtained by consideration of the physical processes affecting the organisms during swimming and accumulation.

The organisms in a convergence zone are stationary relative to the front, but are moving relative to the ambient flow. Thus they are constantly being supplied with 'new' water. This gives a mechanism for enhancing the supply of nutrients or food to the swimmers, enabling the biomass to increase both by the physical effect of accumulation, and the physiological response to increased nutrient or food supply. In the case of phytoplankton, this allows the population to translocate nutrients, creating a biomass far in excess of that attainable by a passive (non-swimming) cell. This situation is commonly seen at tidal fronts, where a single species will often dominate the population, e.g. Gyrodinium aureolum in convergent tidal fronts around the British Isles (Pingree et al. 1975). It was estimated that this bloom contained more nutrients than were available in the water; the present model suggests a mechanism for this nutrient accumulation.

The strategy of holding position relative to the front while nutrients and food are advected past appears to have been adopted by members of higher trophic levels. Wolanski \& Hamner (1988) report columns of whales feeding in a current created by flow past an island. The whales maintained their position relative to the land, while swimming forward in the current, thus exploiting any accumulation of plankton generated in the island wake.

Because they are not under the influence of the ambient vertical velocities, strong swimmers are very likely to create accumulation zones. Weak swimmers may not always create such zones, but their swimming behavior will alter the amount of time they spend in any given region of the flow. Weak floaters are advected toward the pycnocline of a convergent front, and spend more time away from the surface than they would otherwise (Fig. 7). Weak sinkers at a divergent front are buoyed upward by the flow, and so take longer to sink out of the water column (Fig. 6). Such effects may alter the persistence of a population in a given region, and may create a refugium for organisms in certain types of flow.

The model presented above was designed to simulate swimming organisms at fronts, but may equally apply to sinking particulate matter in 2 dimensional flows, benthic larvae in bottom fronts, or flying organisms (e.g. insects) in atmospheric flows. Thus aspects of the model may be useful in understanding the distribution of terrigenous material in coastal regions, the patterns of benthic larval accumulation in strong tidal regions, or the concentrations of locusts in atmospheric fronts.

The most critical assumptions of the model presented are that a 2 -dimensional flow exists, that it is steady, and that the swimming behavior exists. There is ample evidence for directed swimming behavior of organisms in the ocean (particularly sinking), and the assumption of steady flow can probably be relaxed without affecting the basic results (although the solutions become much more complicated). Such matters are certainly worth pursuing. The assumption of a 2-dimensional cross-frontal flow is more problematic. Numerous researchers have hypothesized the existence of a deterministic cross-frontal flow, however most field data emphasize the 3-dimensional, unsteady nature of fronts. The common observation of surface accumulations of floating material at frontal zones, however, 
reinforces the notion of a deterministic cross-frontal convergence. Still, the deeper flows are poorly understood. It is premature, then, to interpret specific data sets quantitatively with this model. However it appears to be a useful heuristic tool for understanding the processes affecting motile organisms at fronts, and interpreting the results of more complete models.

Acknowledgements. I thank Leonard Walstad, Andrew Bennett, Charlie Miller, Larry Small, Dan Kelley and Sharon Roth for helpful comments and criticisms. This work was begun while I was supported by ONR grant N00014-89-J-111 to D. M. Anderson, and completed while I was supported by a UCAR postdoctoral fellowship, and funding from the ONR Coastal Sciences Program grant N0014-90-J-1051 to L. Walstad and J. S. Allen.

\section{LITERATURE CITED}

Batchelor, G. K. (1983). An introduction to fluid dynamics Cambridge University Press, Cambridge

Bauerfeind, E., Elbrachter, M., Steiner, R., Throndsen, J. (1986). Application of laser doppler spectroscopy (LDS) in determining swimming velocities of motile phytoplankton. Mar. Biol. 93: 323-327

Beers, J. R., Stevenson, M. R., Eppley, R. W., Brooks, E. R. (1971). Plankton populations and upwelling off the coast of Peru, June 1969. Fish. Bull. U.S. 69: 859-876

Blasco, D., Estrada, M., Jones, B. H (1981). Short-time variability of phytoplankton populations in upwelling regions - the example of northwest Africa. In: Richards, F. A. (ed.) Coastal upwelling. American Geophysical Union, Washington, D.C., p. 339-347

Chao, S.-Y (1987). Wind-driven motion near inner shelf fronts. J. geophys. Res. 92: 3849-3860

Dengler, A. T (1985). Relationship between physical and biological processes at an upwelling front off Peru, $15^{\circ} \mathrm{S}$. Deep Sea Res. 32: 1301-1315

Evans, G. T., Taylor, F. (1980). Phytoplankton accumulation in Langmuir cells. Limnol. Oceanogr 25: 840-845

Foo, E.-C (1981). A two-dimensional diabatic isopycnal model - simulating the coastal upwelling front. J. phys. Oceanogr. 11: 604-626

Garrett, C. J R., Loder, J. W. (1981). Dynamical aspects of shallow sea fronts. Phil. Trans. R. Soc. Lond. A302: $563-581$

Holligan. P. M. (1981). Biological implications of fronts on the northwest European continental shelf. Phil. Trans. R. Soc Lond. A302: 547-562

Houghton, R. W., Marra, J. (1983). Physical/biological structure and exchange across the thermohaline shelf/slope front in the New York Bight. J. geophys. Res. 88: $4467-4481$

James, I. D. (1978). A note on the circulation induced by a shallow sea front. Estuar. coast. mar. Sci. 7: 197-202

This article was presented by E. \& B. Sherr, Corvallis, Oregon, USA
Jones, B. H., Halpern, D. (1981). Biological and physical aspects of a coastal upwelling event observed during March-April 1974 off northwest Africa. Deep Sea Res. 28A: $71-81$

Kamykowski, D. (1981). The simulation of a southern California red tide using characteristics of a simultaneouslymeasured internal wave field. Ecol. Model. 12: 253-265

Olson, D. B., Backus, R. H. (1985). The concentrating of organisms at fronts: a cold-water fish and a warm-core Gulf Stream ring. J. mar. Res. 43: 113-137

Pingree, R. Forster, G., Morrison, G. (1974). Turbulent convergent tidal fronts. J. mar. biol. Ass. U.K. 54: 469-479

Pingree, R. D., Holligan, P. M., Mardell, G. T (1978). The effects of vertical stability on phytoplankton distributions in the summer on the northeast European shelf. Deep Sea Res. 25: 1011-1028

Pingree, R., Pugh, P., Holligan, P. M., Forster, G. (1975). Summer phytoplankton blooms and red tides in the approaches to the English Channel. Nature, Lond. 258 $672-677$

Richardson, K. (1985). Plankton distribution and activity in the North Sea/Skagerrak-Kattegat frontal area in April 1984 Mar. Ecol. Prog. Ser. 26: 233-244

Seliger, H. H., McKinley, K. R., Biggley, W. H., Rivkin, R. B. Aspden, K. R. H. (1981). Phytoplankton patchiness and frontal regions. Mar. Biol. 61. 119-131

Simpson, J., Allen, C., Morris, N. (1978). Fronts on the continental shelf. J. geophys. Res. 83: 4607-4614

Simpson, J. H., Edlestein, D. J., Edwards, A., Morris, N. C. G. Telt, P. B. (1979). The Islay Front: physical structure and phytoplankton distribution. Estuar. coast. mar. Sci. 9 $713-726$

Simpson, J H., Tett, P. B., Argotte-Espinoza, M. L., Edwards A., Jones, K. J., Savidge, G. (1982). Mixing and phytoplankton growth around an island in a stratified sea. Cont Shelf Res. 1 15-31

Smith, W. O., Heburn, G. W. Barber, R. T., O'Brien, J. J. (1983) Regulation of phytoplankton communities by physical processes in upwelling systems. J. mar. Res. 41: 539-556

Stommel, H. (1949). Trajectories of small bodies sinking slowly through convection cells. J. mar. Res. 8: 25-29

Townsend, D. W., Yentsch, C. M., Parker, C. E., Balch, W. M., True, E. D. (1983). An island mixing effect in the coastal Gulf of Maine. Helgoländer Meeresunters. 36: 347-356

Traganza, E. D., Redalje, D. G. Garwood, R. W. (1987). Chemical flux, mixed layer entrainment and phytoplankton blooms at upwelling fronts in the California coastal zone. Cont. Shelf Res. 7: 89-105

Tyler, M. A., Coats, D. W., Anderson, D. M. (1982). Encystment in a dynamic environment: deposition of dinoflagellate cysts by a frontal convergence. Mar. Ecol. Prog. Ser. 7 : $163-178$

Watanabe, M., Harashima, A. (1986). Interaction between motile phyloplankton and Langmuir circulation. Ecol. Model. 31: 175-183

Werner, F. (1987). A numerical study of secondary flows over continental shelf edges. Cont. Shelf Res. 7: 379-409

Wolanski, E., Hamner, W. M. (1988). Topographically controlled fronts in the ocean and their biological significance. Science 241.177-181

Manuscript first received: September 10,1991

Revised version accepted: February 28, 1992 